\title{
Classified
}

\section{Advertising and Meetings}

\section{ANAESTHETIST - CAMPBELL RIVER, BC}

Anaesthetist required beginning July 1998 at Campbell River and District General Hospital a 70 bed facility serving a community of 40,000 and a secondary referral centre for Northern Vancouver Island. I.C.U. experience and interest desirable. Campbell River is a rapidly growing community with excellent and diverse lifestyle and outdoor recreational opportunities. Please contact:

\section{Dr. Kurt Samer}

Campbell River \& District General Hospital 375 - 2nd Avenue

Campbell River, BC V9W 3V1

Phone: (250) 287-7111 local 319 (Hospital) (250) 923-5599 (Home)

Fax: (250) 286-7073

\section{ANAESTHETIST - TIMMINS, ON}

Timmins and District Hospital has an immediate vacancy for an Anaestherist. Features: new modern building; population 50,000; catchment area 100,000 ; excellent education system; relocation grants; ministry grants; cxcellent recreational facilities. Interested applicants should make inquiries or send a completed curriculum vitae to:

Mr. Paul Gaudreau, Director Administrative and Support Services

Louise Madore, Medical Secretary

Timmins and District Hospital

700 Ross Avenue East

Timmins, ON P4N 8P2

Phone: (705) 267-6330

Fax: (705) 267.631

CLASSIFIED ADVERTISING

As a service to its readers, the Canadian Journal of Anaesthesia is plcased to accept suitable classified advertisements to announce positions or locum tenens available or wanted, postgraduate courses, or other events or items of interest to subscribers. Rates for classified advertising (per insertion): $\$ 1.30$ a word with a minimum charge of $\$ 75.7 \%$ GST (or $15 \%$ HST) is added to Canadian orders. Copy must be received 6 weeks prior to the lst of the month of publication. Ad copy, subject to acceptance by the publisher, should be typed doublespaced and mailed or faxed to:

Canadian Journal of Anaesthesia 1 Eglinton Avenue East, Suite 208 Toronto, Ontario, Canada M4P 3A Tel (416) 480-0602, Fax (416) 480-0320

Specially priced display advertising space is also available to non-commercial advertisers.
FELLOWSHIP POSITIONS IN ANAESTHESIA

The Department of Anaeshesia at the Otrawa General Hospital, University of Ottawa has an opening for a Regional Anaesthesia Fellow commencing July lst, 1998. There are also 2 openings on January lst, 1999 in Chronic Pain Management and Obstetric Anaesthesia. Please contact:

Dr. Dennis Reid, Department of Anaesthesia Ottawa General Hospital

501 Smyth Road

Ottawa, ON KIH 8L6

Phone: (613) 737-8187

Fax: (613) 737.8189

E-mail: dereid@ogh.on.ca

\section{ANAESTHETIST - PRINCE GEORGE, BC}

Prince George Regional Hospital has an immediate opening for a fellowship anaesthetist. This is a permanent full-time position. Prince George Regional Hospital is a 219-bed acute-care hospital with a 10-bed ICU/CCU. It is the referral centre for Northern BC. Surgical services include General, Orthopaedic, Urology, Obstetrics and Gynaecology, ENT, Plastics, Ophthalmology, and Thoracic Surgery supported by six full-time anaesthetists. Interested applicants please send $\mathrm{CV}$ to

Dr. James Lindsay, Head

Department of Anaesthesia

2000 Fifteenth Avenue

Prince George BC V2M 1S2

Phone: (250) 565-2353

Fax: (250) 565-2343

\section{LOCUM POSITION - HAMILTON, ON}

A locum position for an Anaesthetist is being offered at St. Joseph's Hospital, Hamilton, Ontario from January 1, 1999 to December 31, 1999.

St. Joseph's Hospital is a busy tertiary care teaching hospital offering a wide range of surgical procedures and Obstetrics. No Neuro, Cardiac or Neonates. Remuneration is on a percentage of billing basis. Must hold an Ontario license and FRCPC. Please send $\mathrm{CV}$ and references to:

\section{Dr. L. Kahn}

Department of Anaesthesia

St. Joseph's Hospital

50 Charlton Ave. East

Hamilton, ON L8N 4A6

Phone: (905) 522-1155 ext. 3853 (Hospital) (905) 572-9391 (Home)

\section{Meetings - Canada}

Canadian Anaesthetists' Society

55th Annual Meeting

June 12-16, 1998

Westin Harbour Castle, Toronto, Ontario

For information:

Ms. Annette Schilz, Meeting Coordinator

Canadian Anaesthetists' Society

1 Eglinton Ave. East, Suite 208

Toronto, ON M4P 3Al

Phone: (416) 480-0602

Fax: (416) 480-0320

E-mail: cas@multinet.net

WWW: http://www.cas.ca

International Trauma Anesthesia and

Critical Care Society

June 26-July 1, 1998

Montréal, Québec

For information:

ITACCS-NWAS

P.O. Box 2797

Pasco, WA 99302

Phone: 1-800-222-6927

Phone: (509) 547.7065

Fax: (509) 547-1265

E-mail: nwas@owt.com

WWW: http://www.nwas.com/itaces

CAS Atlantic Divisions' Regional Meeting July 10-12, 1998

Charlottetown, PEI

For Information:

Dr. David Shephard

Queen Elizabeth Hospital

Charlottetown, PEI ClA 8T5

Phone: (902) 894-2230

ITACCS - Trauma: A Team Approach

to the Clinical Challenge

July 12-16, 1998

Montréal, Québec

For information:

Paul Hilliard

ITACCS - NWAS

P.O. Box 2797

Pasco, WA 99302

Phone: (509) 547-7065

Fax: (509) 547-1265

E-mail: nwas@owt.com

WWW: http://www.nwas.com/itaccs 
OMA/CAS Annual Meeting

September 11-13, 1998

Peterborough, Ontario

For information:

Dr. R.R. Mann MD, FRCPC

963 Fairbairn Street

Peterborough, ON K9K 1 B8

Phone: (705) 742-1241

Fax: (705) 745-0400

E-mail: ren.mann@sympatico.ca

International Forum on Paediatric Pain:

Chronic and Recurrent Pain

September 24-27, 1998

White Point Beach, Nova Scotia

For information:

Conventional Wisdom

6469 Liverpool Street

Halifax, NS B3L 1Y4

Phone: (902) 453-4664

Fax: (902) 423-5232

E-mail: katefin@chebucto.ns.ca

WWW: http://is.dal.ca/-pedpain/pedpain.html

Society for Obstetric Anesthesia and

Perinatology: 31st Annual Meeting

May 31-June 3, 2000

Montréal, Québec

For information

SOAP P.O. Box 11086

Richmond, VA 23230-1086

Phone: (804) 282-5051

Fax: (804) 282-0090

E-mail: 75112.2053@i@compuserve.com

International Symposium on Regional

Anesthesia and Pain Medicine

May 31-June 3, 2000

Québec City, Québec

For information

ASRA P.O. Box 11086

Richmond, VA 23230-1086

Phone: (804) 282-0010

Fax: (804) 282-0090

E-mail: SocietyHQ@compuserve.com

Society of Cardiovascular Anesthesiologists

International Symposium

June 1-3, 2000

Québec City, Québec

For information:

SCA P.O. Box 11086

Richmond, VA 23230-1086

Phone: (804) 282-0084

Fax: (804) 282-0090

E-mail: 75112.2053@compuserve.com

12th World Congress of Anaesthesiologists June 4-9, 2000

Palais des Congrès, Montréal, Québec

For information.

Congress Secretariat

c/o Events International Meeting Planners Inc.

759 Victoria Square, Suite 300

Montréal, Québec, Canada H2Y 2J7

Phone: (514) 286-0855

Fax: (514) 286-6066

E-mail: info@eventsintl.com

WwW: http://www.cas.ca

\section{Other Meetings}

10th European Congress of

Anaesthesiology

June 30-July 4, 1998

Frankfurt/Main, Germany

For information:

MCN Medizinische Congressorganisation

Nürnberg GmbH

Wielandstr.6

D-90419 Nürnberg, Germany

Fax: 49911331204

ITACCS - Trauma: A Team Approach to

the Clinical Challenge

July 20-23, 1998

Cape Cod, Massachusetts

For information:

Paul Hilliard

ITACCS-NWAS

P.O. Box 2797

Pasco, WA 99302

Phone: (509) 547-706

Fax: (509) 547-1265

E-mail: nwas@owt.com

WWW: http://www.nwas.com/itaccs

\section{Horizons in Pulmonary \&}

Critical Care Medicine

August 27-28, 1998

Cleveland, Ohio

For information:

The Cleveland Clinic Foundation

9500 Euclid Avenue

Cleveland, $O H 44195$

Phone: 1-800-762-8173

Fax: (216) 445-9406

ITACCS - Trauma: A Team Approach to

the Clinical Challenge

August 29 -September 10, 1998

Fairbanks, Alaska

For information:

Paul Hilliard

ITACCS-NWAS

P.O. Box 2797

Pasco, WA 99302

Phone: (509) 547-7065

Fax: (509) 547-1265

E-mail: nwas@owt.com

WWW: http://www.nwas.com/itaccs

6th International Congress of

Cardiovascular and Thoracic Anesthesia

September 2-5, 1998

Santiago de Chile, Chile

For infornation:

Society of Cardiovascular Anesthesiologists, USA

P.O. Box 1.1086

Richmond, VA 23230-1086

Phonc: (804) 282-0084

Fax: (804) 282-0090

E-mail: 75112.2053@compuserve.com
European Academy of Anaesthesiology

20th Annual Meeting

September 3-5, 1998

Heraklion, Crete, Greece

For Information:

Triaena Tours

Congress Department

24, Har. Trikoupi Str.

GR 10679 Athens, Greece

Phone: +30-1-3609 511-15

Fax: $+30-1-3607962$

E-mail: triaena3@fnet.gr

11th Annual Congress of the European

Society of Intensive Care Medicine

September 6-9, 1998

Stockholm, Sweden

For information:

ESICM/ESPIC Congress Secretariat

40 Avenue Joseph Wybran

B-1070 Brussels, Belgium

Phone: 32-2-529-58-29

Fax: 32-2-527-00-62

E-mail: esicm @pophost.eunet.be

Controversies in Perioperative Medicine

September 6-11, 1998

Beaune, Burgundy, France

For information:

Julie Ritchie

The Department of Anaesthesia

North York General Hospital

c/o Julie Ritchie

Butterfield and Robinson

Toronto, ON

Phone: 1:800-678-1147

Fax: (416) 864-0541

E-mail: burg98@ibm.net

Vienna International Congress 1998

Anesthesiology \& Preoperative

Management

September 29-October 2, 1998

Vienna, Austria

For information:

Austrian Society of Anesthesiology,

Resuscitation and Intensive Care Medicine

Johann Nepomuk

Vogl - Platz 1/1, A-1180

Vienna, Austria

Society of Neurosurgical Anesthesia and

Critical Care 26th Annual Meeting

October 16, 1998

Orlando, Florida

For information:

SNACC

P.O. Box 11086

Richmond, VA 23230-1086

Phone: (804) 673-9037

Fax: (804) 282-0090

E-mail: 75112.2053@compuserve.com 
Society for Pediatric Anesthesia

12th Annual Meeting

October 16, 1998

Orlando, Florida

For information:

SPA P.O. Box 11086

Richmond, VA 23230-1086

Phone: (804) 282-9780

Fax: (804) 282-0090

E-mail: 75112.2053@compuserve.com

American Society of Anesthesiologists(ASA)

Annual Mecting

October 17.21, 1998

San Diego, California

For Information:

Frank Connell

American Society of Anaesthesiologists

520 N Northwest Highway

Park Ridge, IL 60068-2571

Phone: (847) 825-5586

Fax: (847) 825-1692

11th International Symposium on

Anesthesia and Intensive Care

November 3-6, 1998

Tiberias, Israel

For information

Secretariat, Target Tours

P.O. Box 29041

Tel-Aviv 61290, Israel

Phone: 972-3-5175150

Fax: 972-3-5175155

E-mail: trgt@netvision.net.il.

Survey of Anesthesia

November 6-8, 1998

Cleveland, Ohio

For information:

The Cleveland Clinic Foundation

9500 Euclid Avenue

Cleveland, $\mathrm{OH} 44195$

Phone: 1-800-762-8173

Fax: (216) 445-9406

ITACCS - Trauma: A Team Approach

to the Clinical Challenge

November 14-22, 1998

Costa Rica

For information:

Paul Hilliard

ITACCS-NWAS

P.O. Box 2797

Pasco, WA 99302

Phone: (509) 547-7065

Fax: (509) 547-1265

E-mail: nwas@owt.con

WWW: http://www.nwas.com/itaces
Pain Management - Toward 2000

November 17-21, 1998

Eilat, Israel

For information:

Israel Pain Association

Prof. D. Niv

4 Bezalel Street

52521 Ramat-Gan, Israel

Phone: 972-3-610-4848

Fax: 972-3-752-1577

WWW: http://www.salus.it/PMT2000

WWW: http://wipain.org

ITACCS - Trauma: A Team Approach to the Clinical Challenge

December 5-12, 1998

Puerto Plata, Dominican Republic

For information:

Paul Hilliard

ITACCS-NWAS

P.O. Box 2797

Pasco, WA 99302

Phone: (509) 547-7065

Fax: (509) 547-1265

E-mail: nwas@owt.com

WWW: http://www.nwas.com/itaccs

European Society of Obstetric

Anaesthesiology

5th International Congress

December 10-12, 1998

Oulu, Finland

For information:

Dr. Pirjo Ranta

Oulu University Hospital

Department of Anaesthesiology

Kajaanintie 50

SF-90220 Oulu, Finland

Phone: 358-8-3152011, ext. 2287

Fax: 358-8-3155577

17th Annual Symposium Clinical Update

in Anesthesiology and Advances in

Techniques of Cardiopulmonary Bypass

January 16-23, 1999

St. Thomas, US Virgin Islands

For information.

Helen Phillips

Mount Sinai Medical Center

1 Gustave L. Levy Place

P.O. Box 1010

New York, NY 10029-6574

Tel: (212) 241-7630

Fax: (212) 426-2009 73rd Clinical and Scientific Congress of the International Anesthesia Research Society (IARS)

March 12-16, 1999

Los Angeles, California

For information:

LARS

2 Summit Park Drive, Suite 140

Cleveland, $\mathrm{OH}$ 44131-2553

Phone: (216) 642-1124

Fax: (216) 642-1127

Anaesthesia \& Intensive Care Conference

June 16-20, 1999

Sun City, South Africa

For information:

SASA99 Congress

P.O. Box 2808

Cramerview 2060, South Africa

Phone: 2711-788-1181

Fax: 2711-788-1775

E-mail: sasa@anaesthetist.com

WWW: http://www.anaesthetist.con

9th World Congress on Pain

August 22-27, 1999

Vienna, Austria

For information:

IASP Secretariat

909 NE 43rd Street, Suite 306

Seattle, WA 98105

Phone: (206) 547-6409

Fax: (206) 547-1703

E-mail: LASP@locke.hs.washington.edu

Continuing Education Committee

Anaesthetists of New Zealand

August 25-28, 1999

Wellington, New Zealand

For information:

Dr. Graham J. Sharpe

Department of Anaesthesia

Wellington Hospita

Capital Coast Health Limited

P.O. Box 7902

Wellington South, New Zealand

Phone: 64-4-385-5920

Fax: 64-4-385-5557

E-mail: woprsb@mash.wnhealth.co.nz 